\title{
THE CORWIN-GREENLEAF PARITY RESULT FAILS FOR COMPLETELY SOLVABLE LIE GROUPS
}

\author{
RONALD L. LIPSMAN
}

(Communicated by Jonathan M. Rosenberg)

The results of Corwin-Greenleaf [1] on orbital decompositions of induced (and restricted) representations for simply connected nilpotent Lie groups have been extended to various kinds of Lie groups $[2,3]$. In addition to explicit formulae for the spectrum and multiplicity, Corwin-Greenleaf were able to obtain some qualitative results on the multiplicity function in the direct integral. These results-in the nilpotent case of course-are:

(1) the multiplicity is always uniformly infinite, or (almost) everywhere finite;

(2) in the finite case, the multiplicity is bounded;

(3) In the finite case, the parity of the multiplicity is constant.

(Actually there is a fourth result-a specific condition which distinguishes between the two situations in (1). But it is known that the condition fails for exponential solvable groups, see [3].)

Many people have speculated as to whether these three results continue to hold for simply connected exponential solvable groups, or if not then in the completely solvable case. In fact, it seems very likely that (1) and (2) are true for exponential solvable groups. (I have an outline of a proof in the completely solvable case.) The purpose of this short note is to indicate that, on the other hand, (3) is false-even for completely solvable groups. The example is deceptively simple, but has apparently been overlooked by workers in the field.

Example. Let $G$ be the three-dimensional simply connected Lie group whose Lie algebra is spanned by generators $T, X, Y$ satisfying the bracket relations

$$
[T, X]=X, \quad[T, Y]=-Y .
$$

The generic representations of $G$ are given as follows. Let $N=\exp (\mathbf{R} X+\mathbf{R} Y)$, $\chi_{\xi, \eta}(\exp (r X+s Y))=e^{i(r \xi+s \eta)}, \pi_{\xi, \eta}=\operatorname{Ind}_{N}^{G} \chi_{\xi, \eta}$. For $\xi^{2}+\eta^{2} \neq 0, \pi_{\xi, \eta}$ is irreducible, and $\pi_{\xi, \eta} \cong \pi_{\xi^{\prime}, \eta^{\prime}} \Leftrightarrow \xi \eta=\xi^{\prime} \eta^{\prime}, \operatorname{sgn}(\xi)=\operatorname{sgn}\left(\xi^{\prime}\right), \operatorname{sgn}(\eta)=\operatorname{sgn}\left(\eta^{\prime}\right)$.

Received by the editors July 21,1989 and, in revised form, July 28, 1989.

1980 Mathematics Subject Classification (1985 Revision). Primary 22E27.

Research supported by NSF grant \#8700551A02. 
Let $H=\exp \mathbf{R}(X+Y)$. Define $\chi_{1} \in \widehat{H}$ by $\chi_{1}(\exp t(X+Y))=e^{i t}$. Then (*) $\quad \operatorname{Ind}_{H}^{G} \chi_{1}=\operatorname{Ind}_{N}^{G} \operatorname{Ind}_{H}^{N} \chi_{1}=\operatorname{Ind}_{N}^{G} \int_{\mathbf{R}}^{\oplus} \chi_{\xi, 1-\xi} d \xi=\int_{\mathbf{R}}^{\oplus} \pi_{\xi, 1-\xi} d \xi$.

The multiplicity in formula $(*)$ is accounted for by the equivalences amongst the representations $\pi_{\xi, 1-\xi}, \xi \in \mathbf{R}$. Indeed for $(\xi, \eta) \in \mathbf{R}^{2}, \xi+\eta=1$, the multiplicity $m(\xi, \eta)$ of the representation $\pi_{\xi, \eta}$ in formula (*) is given by

$$
m(\xi, \eta)= \begin{cases}0 & \xi \eta>\frac{1}{4} ; \text { or } 0<\xi \eta<\frac{1}{4} \text { and } \operatorname{sgn}(\xi)<0 \\ 1 & \xi \eta<0 \\ 2 & 0<\xi \eta<\frac{1}{4} \text { and } \operatorname{sgn}(\xi)>0\end{cases}
$$

The result is illustrated in the diagram below. The diagram also suggests the following interpretation of the phenomenon. The Corwin-Greenleaf result fails for $G$-a locally algebraic group. However it does hold for the algebraic hull of $G$. It is conceivable that the parity results always holds for real algebraic type $E$ solvable groups.

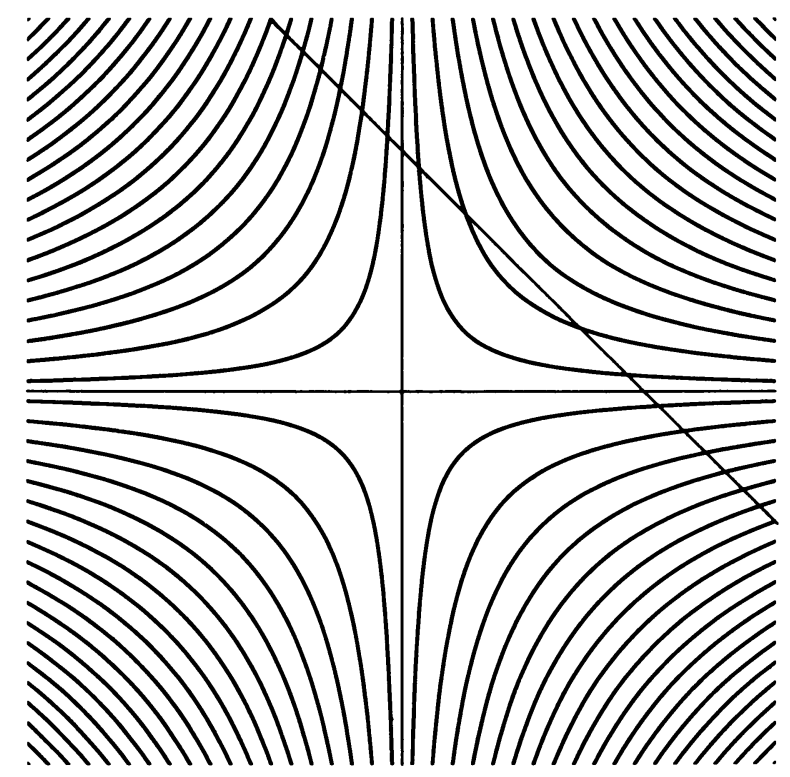

\section{REFERENCES}

1. L. Corwin and F. Greenleaf, Direct integral decompositions and multiplicities for induced representations of nilpotent Lie groups, Trans. Amer. Math. Soc. 304 (1987), 549-583.

2. H. Fujiwara and S. Yamagami, Certaines representations monomiales d'un groupe de Lie résoluble exponentiel, Adv. Stud. Pure Math. 14 (1988), 153-190.

3. R. Lipsman, Orbital parameters for induced and restricted representations, Trans. Amer. Math. Soc. 313 (1989), 433-473.

Department of Mathematics, University of Maryland, College Park, Maryland 